\title{
Failure Analysis and Reinforcement of 3D-printed Concrete Envelope Structure Based on Phase-field Theory (PFT)
}

\author{
Fu Xiao ${ }^{1, *}$, Wu Jie ${ }^{1}$ \\ 1. School of Civil Engineering and Transportation, Hebei University of Technology, Tianjin 300401
}

\begin{abstract}
The permanent 3D-printed concrete envelope formwork can realize the personalized printing of special-shaped envelope structures efficiently with a high degree of automation and obvious advantages. This paper calculated the load-bearing characteristics of the 3D-printed concrete envelope formwork and analyzed the influence of steel supports on its load-bearing characteristics based on PFT. The study showed that the structure would have the highest bearing capacity when the position of steel support reinforcement was near the bisected point of the structure and close to the resultant point of the external load; steel support reinforcement could increase the bearing capacity of structures by nearly 5 times; compared with the single support, the double support could increase the same by nearly 3 times; and triple support reinforcement played a limited role in the improvement of the bearing capacity of structures.
\end{abstract}

\section{Introduction}

Envelope formwork of different shapes should be made and complicated manual form erecting and removal are required in the construction process of special-shaped reinforced concrete structures. However, special-shaped forms generally have a low reusability rate, which leads to high material consumption. In recent years, the $3 \mathrm{D}$ printing technology can realize the personalized manufacturing of special-shaped concrete envelope structures efficiently with a high degree of automation, simple manufacturing process and obvious advantages.

The demand for reliable mechanical properties of printed concrete is increasing in practical applications in order to meet the needs of buildings and civil engineering [1]. However, 3D-printed concrete forms have a low bearing capacity. Then, how to improve the bearing capacity of envelope structures has become an urgent problem to be solved.

The reinforcement system of concrete structures will form a stable whole after the addition of steel supports, which can avoid the deformation and expansion of the concrete in the pouring process. It is an effective reinforcement method to increase the lateral stiffness of structures ${ }^{[2]}$. The position of the joint between steel supports and the original structure and the quantity of steel supports directly affect the effectiveness of steel support reinforcement in practical applications. However, most current reinforcement schemes are generally designed based on experience with a low degree of quantitative analysis.

Fracture is the main failure mode of 3D-printed concrete forms. The quantitative analysis of their bearing characteristics should consider the simulation of crack initiation and propagation. Existing crack models based on finite element calculation methods can be roughly classified into two types - discrete/ interface crack model based on fracture mechanics ${ }^{[3]}$ and smeared crack model based on damage mechanics [4]. When the discrete/ interface crack model simulates crack propagation, the length of cracks is dependent on the element size and cracks can only grow along the boundary of element grids [5]. In the smeared crack model, excessive strain values will appear in the damage area as damage variables increase, which makes the element stress near the crack greater than the stress in the actual structure, leading to the appearance of false cracks that do not exist in reality in such elements and erroneous calculation results of the phase transition field ${ }^{[6]}$.

Phase-field theory (PFT) is a smeared crack method recently developed, which has attracted wide attention due to its relative ease of numerical calculation of cracks. ${ }^{[7]}$ PFT has unique advantages in the simulation of crack propagation. First, the phase field model reduces the computational complexity associated with singularities and allows finite element analysis of crack propagation without the need for gridding again. At the same time, it uses a scalar field (the so-called phase field) to represent smeared cracks, realizing smooth transition from complete materials to completely broken materials and thereby avoiding the description of cracks as physical discontinuities. Finally, the shape and propagation of cracks depend on the evolution equation of the phase field. Therefore, the realization of the phase field does not require additional work to track the crack surface ${ }^{[8]}$.

Based on the ABAQUS finite element software, this paper conducts a mechanical analysis on 3D-printed envelope structures with PFT and studies the fracture

* Corresponding author: 15956289929@163.com 
resistance of envelope forms and the influence of steel supports on their bearing capacity, providing a reference for the design of 3D-printed envelope structures.

\section{PFT}

PFT uses a scalar field $\phi(x, t) \in[0,1]$ for crack surface dispersion in area $\Omega^{[9,10]}$, which meets the following:

$$
\phi=\left\{\begin{array}{c}
0 \text { material not damaged } \\
1 \quad \text { compelete damage of material }
\end{array}\right.
$$

A typical approximate one-dimensional solution of the phase field is given ${ }^{[9]}$ :

$$
\phi(x)=\exp \left(-|x| / l_{0}\right)
$$

Where, $l_{0}$ is the length scale parameter, which controls the transition zone of the phase field and thus reflects the crack width. The width of the crack area increases with the increase of $l_{0}$. When $l_{0}$ goes to zero, the phase field is manifested as sharp cracks.

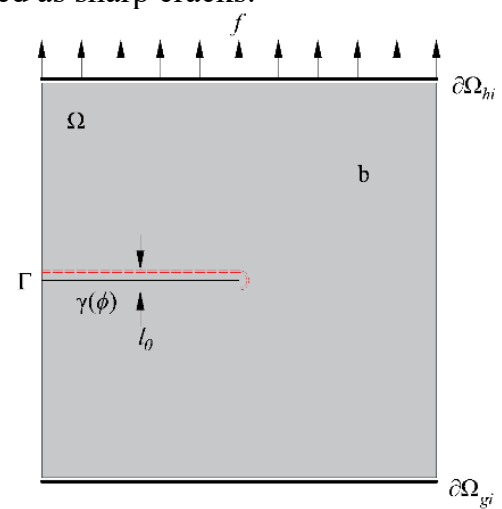

Fig.1 The phase field approximates the cracked surface

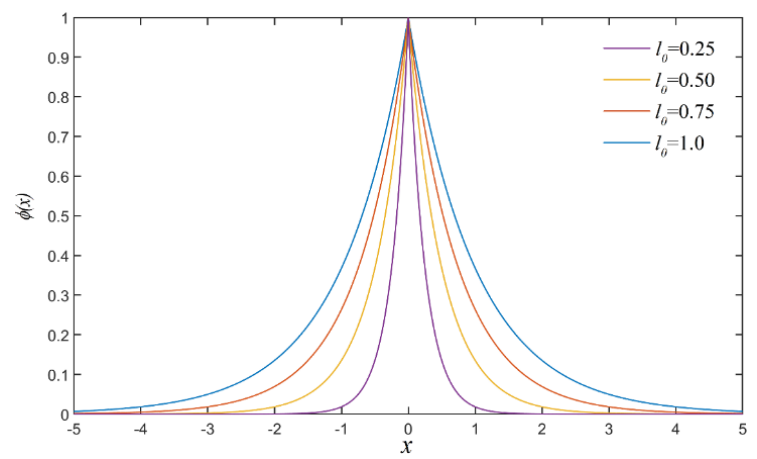

Fig.2 The one-dimensional phase field distribution on the crack

The equation set obtained with PFT must be solved based on the asymptotic iteration method as it is nonlinear. The model above is realized in the software ABAQUS so as to use its built-in nonlinear solver which uses the Newton-Raphson algorithm and the automatic time stepping scheme. In the first iteration of each loading step, the historical field and the phase field are updated by elements in the displacement field and the phase field. The phase field problem is solved according to $H_{n+1}=\psi_{0, n}$. The displacement is solved based on the phase field value of $\phi_{n}$ in the step above. The flowchart in Fig.3 shows the basic iteration process ${ }^{[11]}$.

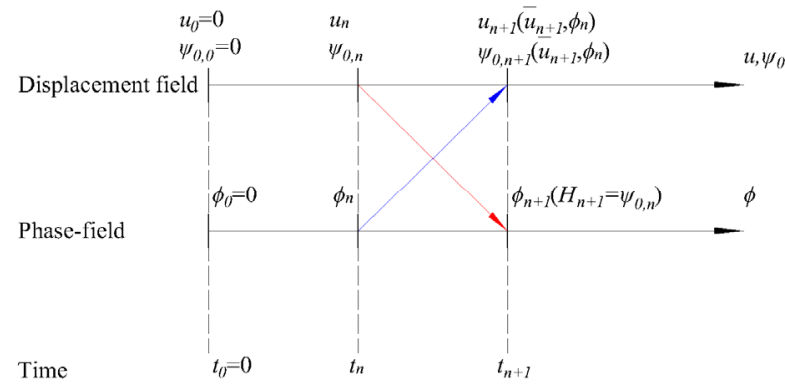

Fig.3 Flowchart of the staggered solution used to implement the coupled displacement phase-field solution in ABAQUS

\section{Failure analysis and reinforcement of 3D-printed envelope structures}

The structure simulated in this section is an envelope structure with multi-layer interlayer interfaces. One side of the structure is subjected to lateral pressure from the poured concrete. Fig. 4 shows its force mechanism and corresponding failure mode ${ }^{[12]}$. A concrete slab, a 3D printer and a concrete pump are preset at the beginning of the construction. The 3D printer first prints the concrete envelope structure at an acceptable height around the preset concrete slab. The height depends on the pressure of the poured concrete. After arrival, the concrete mixer truck delivers the fresh concrete to the pump which delivers the same to the $3 \mathrm{D}$ printer. The $3 \mathrm{D}$ printer then moves among the surrounding envelope walls for printing. After the truck leaves, the 3D printer continues the production of the envelope structure until the next truck arrives. Another concrete pump pours the concrete into the middle part of the envelope structure when the envelope wall reaches the required height.

Next, this paper will explore the failure mode of the structure, observe its weak points, carry out the corresponding steel support reinforcement and discuss the reinforcement scheme. 


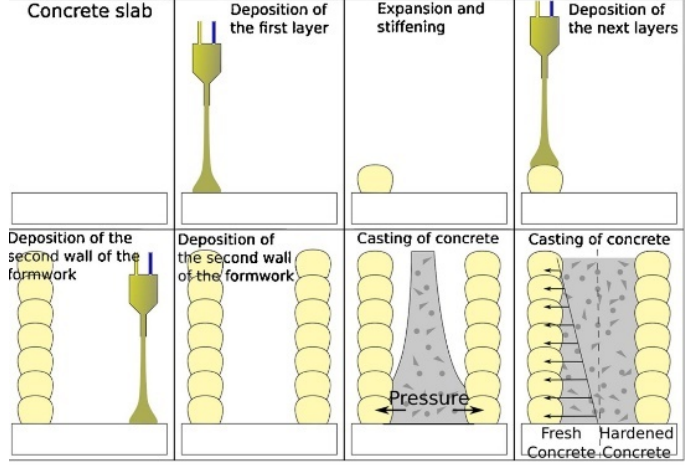

(a) Mechanical principle

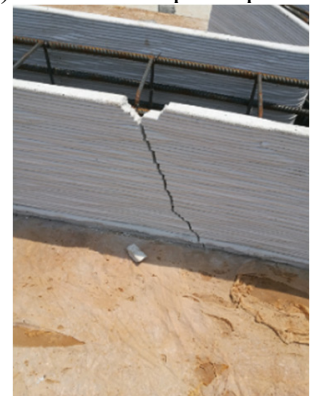

(b) Envelope structural failure

Fig.4 3D-printed concrete envelope structure

\subsection{Establishment of calculation model}

Fig. 5 shows the geometry and boundary conditions of the simplified calculation model for one side of the envelope structure. The bottom of the structure is fixed and the upper end is free. The side is subjected to triangular loads from the poured concrete. The structure has a total height of $1 \mathrm{~m}$ and a width of $120 \mathrm{~mm}$. The height of the concrete layer is $38 \mathrm{~mm}$ and the thickness of the interlayer interface is $2 \mathrm{~mm} .{ }^{[13]}$ As shown in Table 1, the volume weight of the concrete poured on the right side is $\gamma=23128 \mathrm{kN} /$ $\mathrm{m}^{3}$.

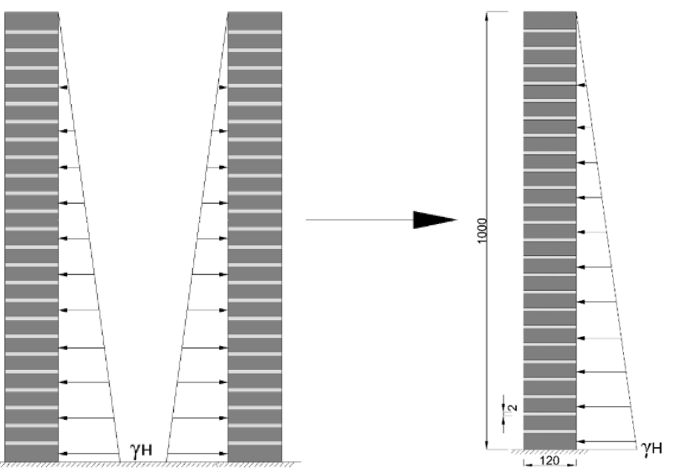

Fig.5 A horizontal 3D-printed concrete envelope structure under side pressure

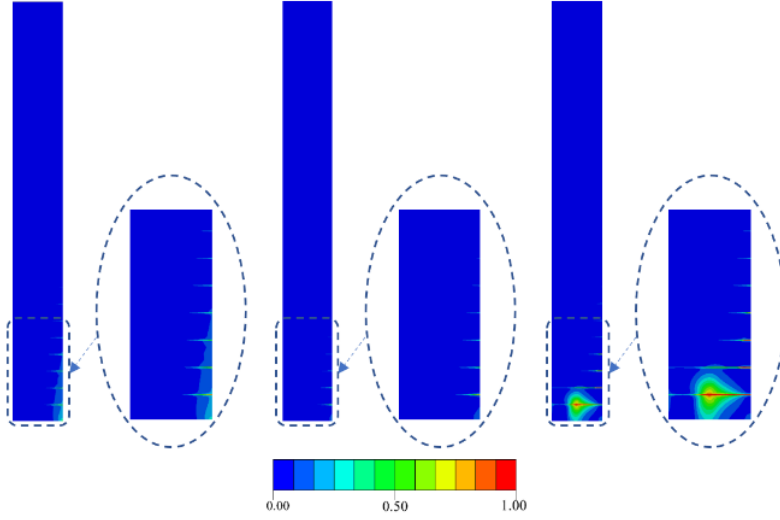

Fig.6 Fracture pattern at different steps by phase filed

The finite element mesh is composed of 107,520 CPS4 elements. Areas around the crack path are detailed with $\mathrm{h}=0.5 \mathrm{~mm}$ as the tessellated mesh size. The side of the structure is loaded with hydrostatic pressure.

Table. 1 Material parameters

\begin{tabular}{cccccc}
\hline & $\mathrm{E}(\mathrm{MPa})$ & $v$ & $\begin{array}{c}\mathrm{G}_{\mathrm{c}}(\mathrm{N} / \\
\mathrm{mm})\end{array}$ & $l_{0}(\mathrm{~mm}$ & $\rho(\mathrm{kg} / \mathrm{m}$ \\
\hline Concrete & 20000 & 0.2 & 0.113 & 1 & 2360 \\
$\begin{array}{c}\text { Interlayer } \\
\text { interface }\end{array}$ & 20000 & 0.2 & 0.01 & 1 & -- \\
\hline
\end{tabular}

Fig.6 shows the complete failure process of the structure. Cracks grow in the interlayer interface near the bottom after the load is applied. However, as cracks spread, only those in the lowest interlayer interface continue to grow until they run through the structure, leading to structural failure. This indicates that the lowest interlayer interface is the most dangerous part of the structure.

\subsection{Influence of steel support position on the bearing capacity of the structure}

The structural failure simulation based on PFT hereinbefore has achieved relatively good results. However, this section will begin to reinforce the envelope structure with steel supports in order to meet the bearing capacity requirements. Steel support positions are set respectively as shown in Fig. 7 so as to explore the influence of the steel support position on the bearing capacity of the envelope structure and further provide a reference for the optimization of 3D-printed envelope structures. Table 2 details specific positions of the steel support. The effect of the steel support is simplified in the calculation model, which is equivalent to a fixed support at the reinforcement point.

Table.2 Steel support reinforcement scheme

\begin{tabular}{cccccc}
\hline Scheme & A & B & C & D & E \\
\hline $\begin{array}{c}\text { Reinforcement } \\
\text { position }\end{array}$ & $1 / 4 \mathrm{H}$ & $1 / 3 \mathrm{H}$ & $1 / 2 \mathrm{H}$ & $2 / 3 \mathrm{H}$ & $3 / 4 \mathrm{H}$ \\
\hline Note: The total height $\mathrm{H}$ of the structure is $1 \mathrm{~m}$.
\end{tabular}

Note: The total height $\mathrm{H}$ of the structure is $1 \mathrm{~m}$. 


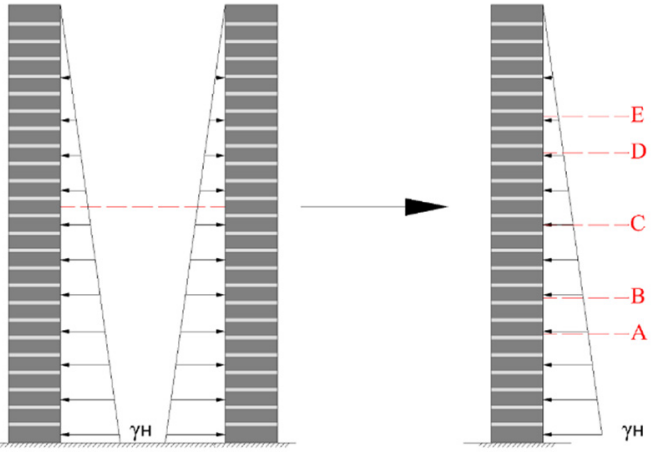

Fig.7 Model simplification and scheme selection

Fig. 8 shows the crack propagation form calculated with PFT in five steel support reinforcement schemes. According to the calculation results, the weak point of the structure is still located in the interlayer interface where cracks are initiated and run through the structure. Under schemes A and B, the structure overturns to the left, cracks are initiated in the upper interface adjacent to the reinforcement point, and the structure is damaged under tension on the right side. Under scheme $\mathrm{C}$, cracks are initiated in the lower interface adjacent to the reinforcement point, and the crack propagation is controlled by shear force. Under schemes D and E, the reinforcement point and the bottom support form a structure similar to a "simply supported beam", cracks are initiated in the mid-span interface, and the structure is damaged under tension on the left side. Cracks are initiated in the lowest interface under both schemes D and $\mathrm{E}$, and the crack propagation is controlled by shear force.
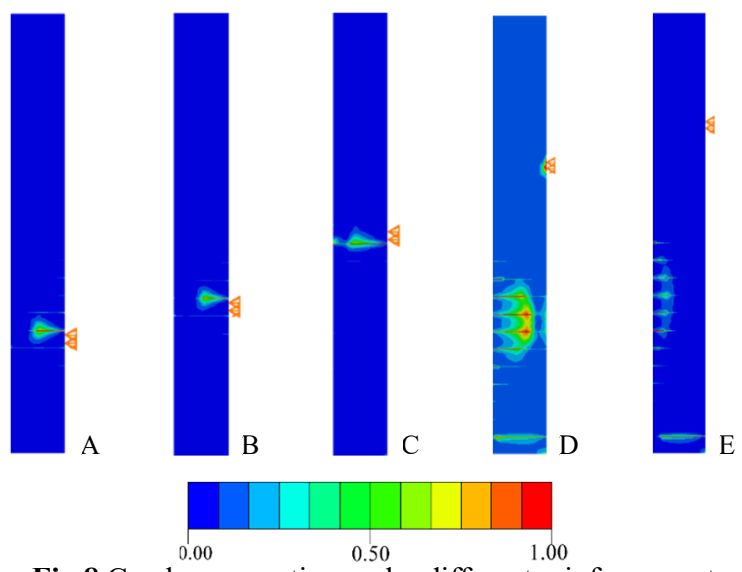

Fig.8 Crack propagation under different reinforcement schemes

Fig.9 shows the deflection curve of the top of the structure under different steel support reinforcement schemes. It can be seen that, compared to the 3D-printed envelope structure without steel support, steel support reinforcement can enhance the overall bearing capacity and stiffness of the structure significantly and effectively. According to the comparison of the five steel support positions, the corresponding structural stiffness and bearing capacity are maximum under scheme $C$, i.e. when the steel support is located at the bisected point of the structure, in which case the bearing capacity of the structure is increased by nearly 5 times.

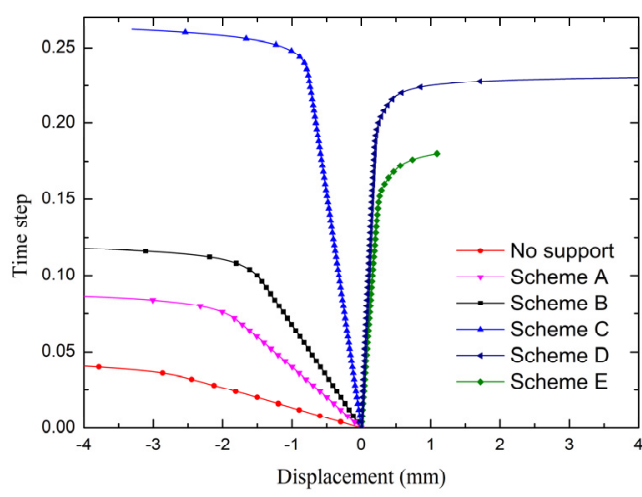

Fig.9 Deflection curve of structure under different reinforcement scheme

\subsection{Influence of steel support spacing on the bearing capacity of the structure}

When the structure has a large size, one steel support is often insufficient to meet the bearing capacity requirements. To further optimize the structure, this section explores the reasonable position and spacing of two steel supports for reinforcement. The calculation model is simplified as shown in Fig. 10. This section puts forward five reinforcement schemes with different spacing around the midpoint of the structure based on the conclusions in 3.2, and compares their advantages and disadvantages. The types of schemes are shown in Table 3 .

Table.3 Double steel support reinforcement scheme

\begin{tabular}{cccccc}
\hline Scheme & A & B & C & D & E \\
\hline $\begin{array}{c}\text { Steel support } \\
\text { spacing (mm) }\end{array}$ & 100 & 200 & 300 & 400 & 500 \\
\hline
\end{tabular}

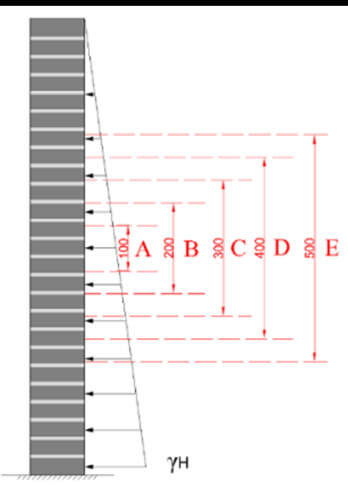

Fig.10 Calculation model under different reinforcement schemes

As shown in Fig. 11, the crack propagation under the five different reinforcement schemes is also different. Under scheme A, cracks are initiated in the central interface of the reinforcement point, and the structure is damaged under tension on the right side. Meanwhile, small cracks appear at the bottom support of the structure. Under schemes B, C and D, cracks are initiated in the interlayer interface on the left and right adjacent to the lower reinforcement point, and the crack propagation is controlled by shear force. At the same time, the lower reinforcement point and the bottom support form a structure similar to a "simply supported beam", and cracks are initiated in the mid-span interface. The structure is 
damaged under tension on the left side. Obvious crack propagation also occurs on the right side of the bottom support of the structure. Under scheme E, the upper and lower reinforcement points form a structure similar to "simply supported beam", cracks are initiated in the midspan interface, and the structure is damaged under tension on the left side.
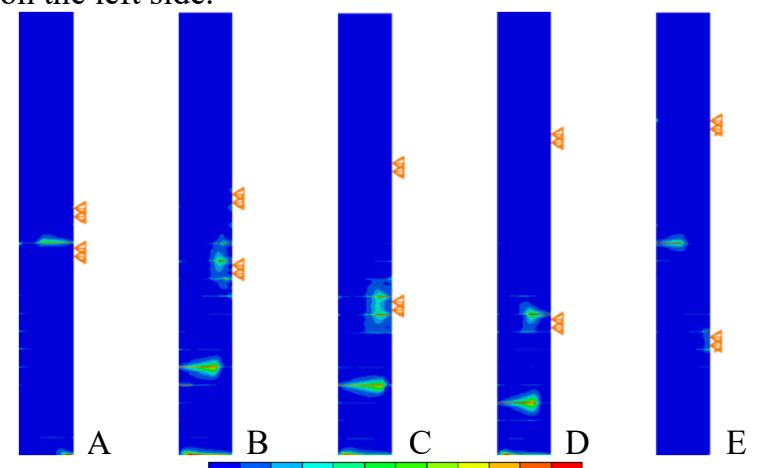

Fig.11 Crack propagation under different reinforcement schemes

As shown in Fig. 12, the comparison of the deflection curves of the top of the structure under the five schemes indicates that double support reinforcement has significantly better effect than single support reinforcement and greatly improves the bearing capacity of the structure. Among the double support reinforcement schemes, D has the best effect, which increases the bearing capacity by nearly three times in comparison with single support reinforcement schemes. Similar to single support reinforcement schemes, double support reinforcement increases the bearing capacity of the structure while enhancing the structural stiffness.

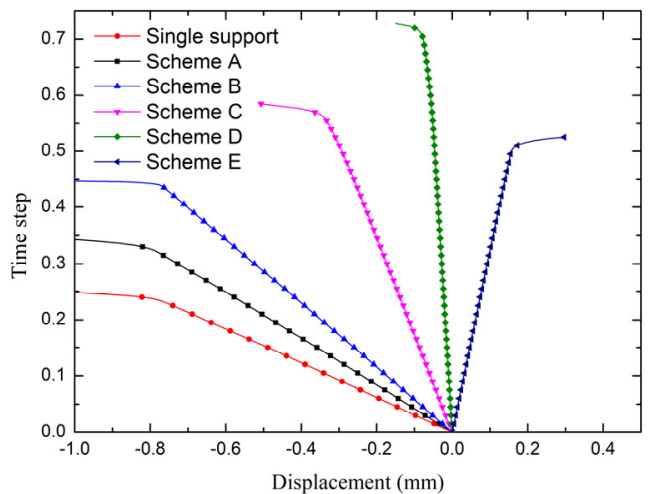

Fig.12 Deflection curve of structure under different reinforcement schemes

Double support reinforcement has a good effect. Next, two triple support reinforcement schemes will be proposed based on the number of steel supports to discuss the reasonable number of steel supports for the structure. The simplified model of the proposed schemes is shown in Fig. 13. Another steel support is added respectively at the two weak points of the structure based on scheme D in 3.3 .
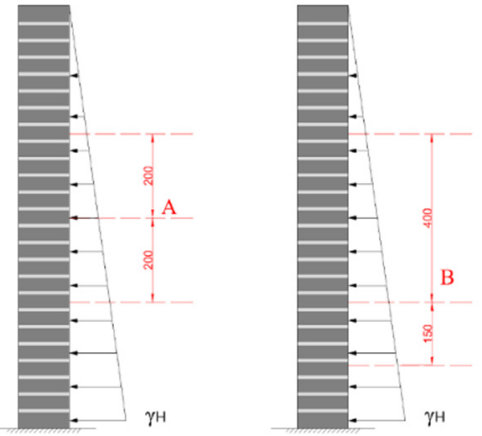

Fig.13 Calculation model under different reinforcement schemes
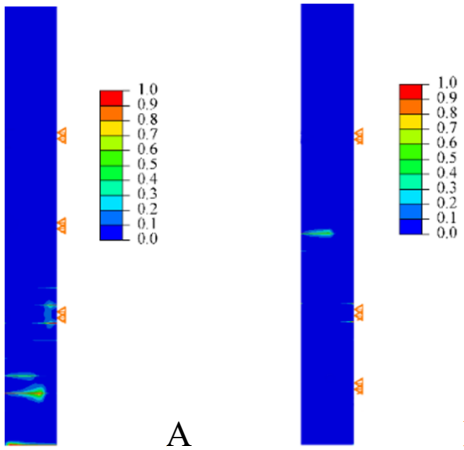

Fig.14 Crack propagation under different reinforcement schemes

Fig. 14 shows the crack propagation mode of the structure under the two schemes. Under scheme A, the lowermost reinforcement point and the bottom support form a structure similar to a "simply supported beam", cracks are initiated in the mid-span interface, and the structure is damaged under tension on the left side. Meanwhile, small cracks spread from the right side of the bottom support. Under scheme B, the upper reinforcement point and the medium reinforcement point form a structure similar to a "simply supported beam", cracks are initiated in the mid-span interface, and the structure is damaged under tension on the left side.

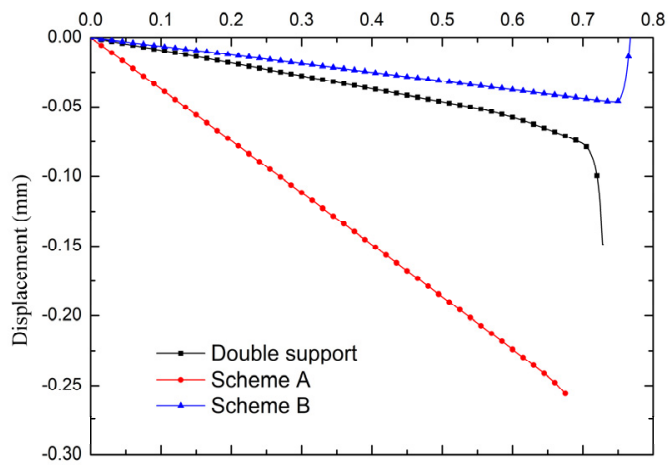

Fig.15 Deflection curve of structure under different reinforcement schemes

The deflection curve of the top of the structure is shown in Fig. 15. The comparison of the structural response under each scheme indicates that the bearing capacity of the structure under scheme A remains almost unchanged compared with the double support scheme; under scheme B, the bearing capacity has a slight increase. 
Considering that the triple support structure has no significant improvement in the bearing capacity compared to the double support structure, the reasonable number of steel supports is 2 for the structure.

\subsection{Structural optimization}

The location of double support reinforcement was selected with reference to the single support structure in the above. This section will discuss the reasonable position of double support reinforcement and determine the final structural optimization plan. Three different reinforcement schemes are proposed, and the spacing of steel supports is $400 \mathrm{~mm}$ under the three schemes. The simplified model is shown in Fig. 16.

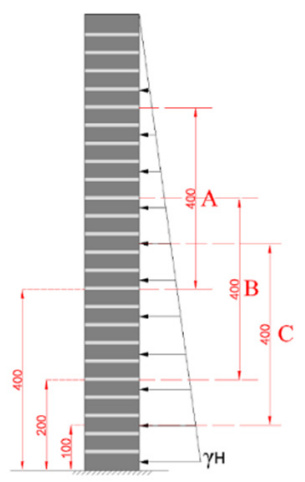

Fig.16 Calculation model under different reinforcement schemes

The crack propagation mode captured is shown in Fig. 17. The crack propagation mode is different under different reinforcement schemes. Under scheme A, there are cracks controlled by bending moment and shear force at the same time. Under scheme B, the structure only has crack propagation in the midspan of the "simply supported beam". Under scheme $\mathrm{C}$, there are only cracks located at the upper reinforcement point and controlled by shear force.

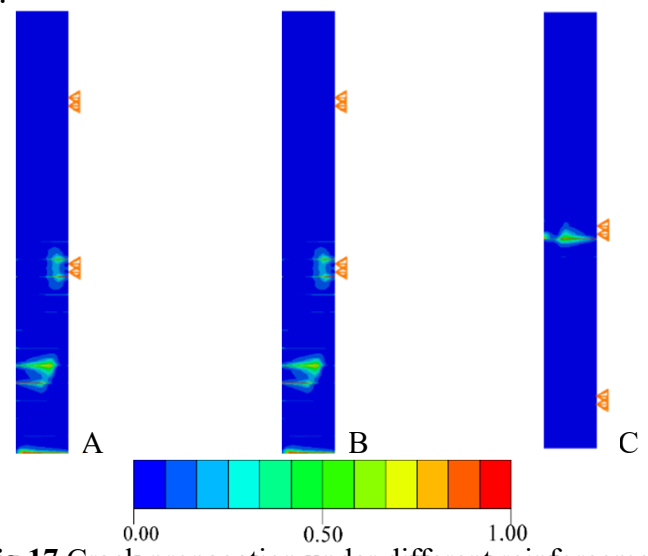

Fig.17 Crack propagation under different reinforcement schemes

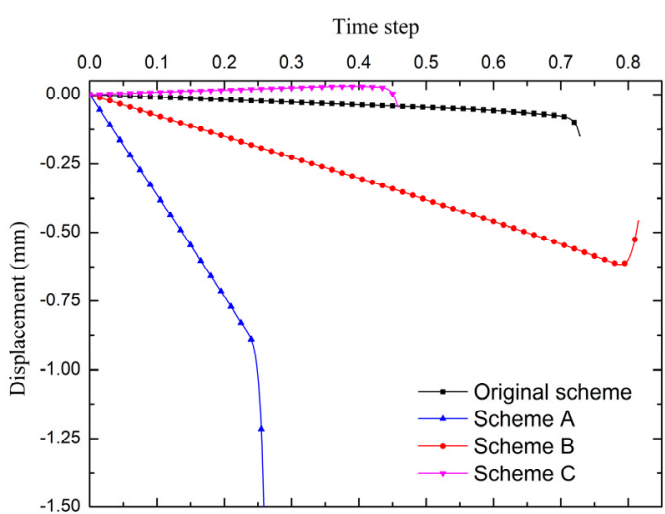

Fig.18 Deflection curve of structure under different reinforcement schemes

The deflection curves under the three schemes are observed (Fig. 18). It can be seen that the bearing capacity of the structure under scheme B has improved to a certain extent compared with scheme D discussed in 2.3. In such case, the reinforcement scheme is the best one among the three schemes. The closer the reinforcement position is to the resultant point of load, the higher the bearing capacity of the structure and the better the structural optimization effect.

\section{Conclusion and outlook}

The 3D-printed concrete envelope formwork has the advantages of high flexibility and no requirement for removal. This paper simulated the fracture of the 3Dprinted concrete envelope formwork structure and studied its bearing capacity and the influence of steel supports on its bearing capacity based on PFT, and the following conclusions are made:

1. PFT can simulate the crack-interface interaction of the permanent 3D-printed envelope formwork under load and calculate its load-bearing characteristics reasonably.

2. Steel supports can improve the bearing capacity of the structure effectively. The reinforcement position should be located around the bisected point of the structure and close to the resultant point of the external load.

3. For the structure in this paper, steel support reinforcement can increase its bearing capacity by nearly 5 times. Compared with the single support, the double support can increase the bearing capacity of structures by nearly 3 times. However, triple support reinforcement plays a limited role in the improvement of the bearing capacity of structures. Therefore, the appropriate number of steel supports should be selected according to the actual size of the structure to avoid material waste.

It is a direction for further study to consider the dynamic effect in the concrete grouting process and study the optimal design of 3D-printed envelope formworksteel support structures under the action of dynamic load with back analysis methods such as topological optimization to improve the bearing capacity of the formwork, reduce the number of steel supports and simplify the construction process. In addition, the 3Dprinted envelope structure has strong three-dimensional 
characteristics. The simplified two-dimensional simulation has certain limitations. Therefore, the threedimensional structural characteristic analysis is also the next research direction.

\section{Acknowledgments}

This paper is one of the phased achievements of the National Natural Science Foundation of China (41807277).

\section{Reference}

1. Wolfs, R.J.M., F.P.Bos, and T.A.M.Salet, Hardened properties of 3D printed concrete: The influence of process parameters on interlayer adhesion[J].Cement and Concrete Research, 2019. 119: p.132-140.

2. Fan Surong. Experimental study on reinforcement of reinforced concrete frame structures with steel supports [D]. Nanjing Tech Technology, 2002.

3. Ngo, D. and A.Scordelis.Finite Element Analysis of Reinforced Concrete Beams.1967.

4. Rashid, Y.R.J.N.E. and Design, Ultimate strength analysis of prestressed concrete pressure vessels.1968. 7: p.334-344.

5. Liu Quansheng, Liu Xuewei. Research on key issues of rock fracture propagation and evolution under multi-field coupling [J]. Rock and Soil Mechanics, 2014, 35(02):305-320.

6. Comi, C., et al., An extended FE strategy for transition from continuum damage to mode I cohesive crack propagation[J]. International Journal for Numerical and Analytical Methods in Geomechanics, 2007. 31(2): p.213-238.

7. Bourdin, B., et al., Numerical experiments in revisited brittle fracture[J]. Journal of the Mechanics and Physics of Solids ,2000. 48(4): p.797-826.

8. Borden, M.J., et al., A phase-field description of dynamic brittle fracture[J]. Computer Methods in Applied Mechanics and Engineering, 2012. 217-220: p.77-95.

9. Miehe, C., M. Hofacker, and F. Welschinger, a phase field model for rate-independent crack propagation: Robust algorithmic implementation based on operator splits[J]. Computer Methods in Applied Mechanics and Engineering, 2010. 199(45): p.27652778.

10. Miehe, C., F. Welschinger, and M. Hofacker, Thermodynamically consistent phase-field models of fracture: Variational principles and multi-field FE implementations[J]. International Journal for Numerical Methods in Engineering, Vol.83.2010.1273-1311.

11. Molnár, G. and A. Gravouil, 2D and 3D Abaqus implementation of a robust staggered phase-field solution for modeling brittle fracture[J]. Finite Elements in Analysis and Design, 2017. 130: p.27-38.

12. Furet, B., P. Poullain, and S. Garnier, 3D printing for construction based on a complex wall of polymerfoam and concrete[J].Additive Manufacturing, 2019. 28: p.58-64.

13. Wu, J.-Y. and V.P. Nguyen, A length scale insensitive phase-field damage model for brittle fracture[J]. Journal of the Mechanics and Physics of Solids, 2018. 119: p.20-42. 